\title{
Vitamin D deficiency - a potential risk factor for sepsis development, correlation with inflammatory markers, SOFA score and higher early mortality risk in sepsis
}

\author{
Olejarova $\mathrm{M}^{1,2}$, Dobisova $\mathrm{A}^{3}$, Suchankova $\mathrm{M}^{1}$, Tibenska $\mathrm{E}^{4}$, Szaboova $\mathrm{K}^{4}$, Koutun $\mathrm{J}^{3}$, \\ Vlnieskova $\mathrm{K}$, Bucova $\mathrm{M}^{1}$ \\ Institute of Immunology, Faculty of Medicine, Comenius University, Bratislava, Slovakia. \\ maria.bucova@fmed.uniba.sk
}

\begin{abstract}
OBJECTIVES: Sepsis is a life-threatening organ dysfunction generated due to the dysregulation of the immune response to infection. The aim of this study was to highlight the role of vitamin $D$ in sepsis and non-infectious SIRS (systemic inflammatory response syndrome) and to find correlation of vitamin D levels with inflammatory markers, severity of the disease, and association with the 7th and 28th survival rate of patients.

METHODS: We investigated 32 patients (21 men, 11 women) admitted to an intensive care unit with both SIRS and sepsis. Blood was taken within 24 hours after admission. Plasma levels of 25(OH)D, sTREM-1, CRP, presepsin and procalcitonin were investigated.

RESULTS: Patients with sepsis had lower levels of 25(OH)D $(n=25)$ than SIRS patients $(n=7 ; p=0.0032)$. Significantly lower levels of $25(\mathrm{OH}) \mathrm{D}$ were found also in patients, who did not survive the 7 th $(p=0.0076)$ and 28 th day $(p=0.0338)$ of hospital care compared to 7 th, resp. 28th day survivors. We revealed a negative correlation between the levels of 25(OH)D and inflammatory markers CRP $(p=0.0003)$, presepsin $(p=0.0032)$ and STREM-1 $(p=0.0065)$ in all SIRS/sepsis patients and clinical condition (SOFA score; $p=0.0385$ ).

CONCLUSION: Our results showed that vitamin $D$ deficiency predisposed to the development of sepsis, negatively correlated with CRP, presepsin, STREM-1 and SOFA score and their levels associates with both 7th and 28th days survival of patients (Tab. 5, Ref. 64). Text in PDF www.elis.sk.

KEY WORDS: procalcitonin, presepsin, sepsis, SIRS, STREM-1, vitamin D.
\end{abstract}

\section{Introduction}

Sepsis was known since the time of Hippocrates (460-377 BC) without exact information regarding its pathogenesis (1). According to the Third International Consensus Definitions for Sepsis and Septic Shock (Sepsis-3) in 2016, sepsis was defined as a life-threatening organ dysfunction caused by a dysregulated host's immune response to infection (2). This severe complex clinical syndrome is a major cause of admission to the intensive care unit (ICU) and death from infectious diseases $(2,3)$. It is estimated, that sepsis is the leading cause of mortality and critical

${ }^{1}$ Institute of Immunology, Faculty of Medicine, Comenius University, Bratislava, Slovakia, ${ }^{2}$ Institute of Physiology, Faculty of Medicine, Comenius University, Bratislava, Slovakia, ${ }^{3} 1$ st Department of Anesthesiology and Intensive Care Medicine, Faculty of Medicine, Comenius University and University Hospital, Bratislava, Slovakia, and ${ }^{4}$ Department of Immunology, Medirex Ltd., Bratislava, Slovakia

Address for correspondence: M. Bucova, MD, PhD, Institute of Immunology, Faculty of Medicine, Comenius University Bratislava, Odborarske nam 14, SK-813 72 Bratislava, Slovakia.

Phone: +421.2 .59357351$

Acknowledgements: This work was supported by the grant UK/402/2015, $\mathrm{UK} / 451 / 2016, \mathrm{UK} / 346 / 2017$ and UK/279/2018. illness worldwide $(4,5)$. Even with optimal medical care, mortality rates in severe sepsis increase to around $50 \%$ (6).

A wide range of pathogens could cause such potentially lethal responses leading to single or multiple vital organ failure. The non-infectious systemic inflammatory response syndrome (SIRS) can be triggered by diverse forms of injury including burns, ischemia, autoimmune diseases, surgery and traumatic injuries $(7,8)$. The prevalence of SIRS is very high, affecting one-third of all in-hospital patients and more than $50 \%$ of all ICU patients $(6,9)$.

The development of systemic inflammation starts with the activation of the mechanisms of natural immunity. Pattern recognition receptors (PRR) on the surface of monocytes/macrophages, vascular endothelial cells and other stromal cells recognize and bind pathogen associated molecular patterns (PAMPs) on the surface of microorganisms or their DNA or RNA during infection. These PRR recognize also danger/damage-associated molecular patterns (DAMPs) - "alarmins" of endogenous origin. These "signals of threatening" - e.g. high-mobility group box 1 (HMGB1) protein, histones, heat shock proteins (HSPs), fibrinogen, heparin - sulfate and other substances are released after trauma, burn, surgical intervention, cell and tissue injury, cell necrosis, etc. The binding of PAMPs and DAMPS on PRR results in the activation of inflamasomes and production of proinflammatory cytokines IL- 
$1 \beta$ and IL-18 and development of inflammation (10-14). It leads to an auto-amplifying production of numerous pro-inflammatory molecules including TNF- $\alpha$, IL-1 $\beta$, IL-2, IL-6, IL-8, HMGB1, TREM-1 (triggering receptor expressed on myelocytes) and IFN- $\gamma$ in a so-called "cytokine storm" (15-17). To avoid the negative side effects of an exaggerated inflammatory reaction and hyperactivated Th1 immune response, almost simultaneously (after 24 hours) with the development of the inflammatory response, a counter-regulatory anti-inflammatory reaction develops (compensatory anti-inflammatory response syndrome - CARS) and Th2 immune response is activated (IL-4, IL-10) $(8,9)$. Loss of control over this balance may lead to organ dysfunction or failure due to SIRS, or immune suppression, even an immune paralysis associated with fatal polymicrobial sepsis may be the result of severe contra regulatory anti-inflammatory response syndrome (previous exaggerated CARS) development (18).

There are clinical scores, such as the Sequential (Sepsis-related) Organ Failure Assessment (SOFA), bedside quickSOFA (qSOFA) $(2,19)$ and the Acute Physiology And Chronic Health Evaluation II (APACHE II) (20), used for organ dysfunction representation and SIRS severity and patient mortality risk evaluation.

Vitamin D (VD) is a neuro-hormone regulating bone calciumphosphate homeostasis, but plays also a major role in extra-skeletal metabolic processes, such as glucose metabolism, and in many aspects of cellular functions and immunomodulation (21-24). It has a direct effect on the function of both innate and adaptive immunity via VD receptor expressed on several immune cells (25-28).

Anti-inflammatory effect of VD in human T cells is partially mediated by inhibitory effect on NFKB (29). VD also participates in the shifting of Thelper (Th) cell response from Th1 (specific cell mediated immunity accompanied by inflammation) to Th2 (specific humoral immunity). Inhibiting the production of Th1 cytokine IFN- $\gamma$ and increasing production of Th 2 cytokines IL-4, IL-5 and IL-10, VD may limit the potential tissue damage associated with excessive Th1 cellular immune responses and hyperinflammation (ongoing also in SIRS or sepsis) (30, 31). Furthermore, VD can suppress IL-17 production by Th17 cells and has been proven to promote self-tolerance $(22,32,33)$.

VD deficiency is associated with various disorders such as: diabetes, infections, myocardial infarction, autoimmune disease, chronic obstructive pulmonary disease, tuberculosis, and excess mortality in the general population (34-36).

25-hydroxyvitamin D $(25(\mathrm{OH}) \mathrm{D})$ is the major circulating form of VD that has a half-life of approximately 2-3 weeks. It is a summation of both VD intake and VD produced from sun exposure and it is just that VD metabolite used to determine whether a patient is VD deficient, sufficient or intoxicated $(37,38)$. There is no absolute consensus about the normal range for $25(\mathrm{OH}) \mathrm{D}$, but most experts now agree that VD deficiency should be defined as a $25(\mathrm{OH}) \mathrm{D}$ level of $<20 \mathrm{ng} / \mathrm{mL}$ (50 nmol/L). VD insufficiency is classified as a serum $25(\mathrm{OH}) \mathrm{D}$ level between 20 and $29 \mathrm{ng} / \mathrm{mL}$ $(50-74 \mathrm{nmol} / \mathrm{L})$. The preferred level for $25(\mathrm{OH}) \mathrm{D}$ is now recommended to be $>30 \mathrm{ng} / \mathrm{mL}(75 \mathrm{nmol} / \mathrm{L})(37-40)$.

The aim of our study was to find the association of VD plasma levels with inflammatory markers, clinical condition of patients expressed by SOFA and APACHE II score and the 7th and 28th day mortality of SIRS/sepsis patients.

\section{Subjects and methods}

We investigated 32 adult patients ( 21 men, 11 women, mean age $59.313 \pm 13.104$ years) admitted to the intensive care unit (ICU) of the 1st Department of Anesthesiology and Intensive Care Medicine, Faculty of Medicine, Comenius University and University Hospital Bratislava, Slovakia. In our study, adult patients with suspected or running systemic inflammatory response (sepsis, septic shock or non-infectious SIRS) and with supposed hospitalization length more than 24 hours were enrolled (Tab. 1). Exclusion criteria contained age below 18 years, diagnosis of primary immunodeficiency disease or immunosuppressive therapy in history. Patients in terminal stage with a presumed death within 24 hours from admission to ICU were also excluded.

All patients were examined by physicians and routine laboratory investigations, including complete and differential blood count and inflammatory markers, blood culture and site specific culture as indicated, were performed. Baseline clinical data collected were sex, age, body mass index (BMI), comorbidities, APACHE II score and SOFA score in the first 24 hours, duration of mechanical ventilation, length of the ICU stay and 7th and 28th day mortality. Patients were followed up until their discharge from the ICU or death.

For purpose of this study, $10 \mathrm{~mL}$ of blood was taken within 24 hours from admission to the ICU, $5 \mathrm{ml}$ into a tubes with EDTA (for DNA and plasma isolation) and $5 \mathrm{~mL}$ into a tube without anti-coagulants (for isolation of serum). Samples were centrifuged and serum and plasma were stored at $-80{ }^{\circ} \mathrm{C}$ until the end of the study, when all of the samples were analyzed for each marker as a single batch.

Various pro-inflammatory cytokines and inflammatory markers were investigated, including plasma level of sTREM-1 by Enzyme-linked Immunosorbent Assay (Human sTREM-1 Elisa kit; Cloud-Clone Corp., Houston, USA), all in strict accordance with manufacturer's instructions. The plasma concentrations of presepsin (sCD14-ST) were investigated by a PATHFAST Presepsin chemiluminescent enzyme immunoassay (CLEIA; Mitsubishi

Tab. 1. Characteristics of investigated groups of patients.

\begin{tabular}{lcccc}
\hline & All patients & SIRS & Sepsis & Septic shock \\
\hline Number & 32 & 7 & 11 & 14 \\
Mean age (years) & $59.3 \pm 13.1$ & $59.5 \pm 20.7$ & $58.1 \pm 12.5$ & $60.1 \pm 9.3$ \\
Sex (men/women) & $21 / 11$ & $3 / 4$ & $9 / 2$ & $9 / 5$ \\
VD severe deficiency & 16 & 1 & 4 & 11 \\
VD deficiency & 13 & 4 & 6 & 3 \\
VD insufficiency & 2 & 1 & 1 & 0 \\
VD sufficiency & 1 & 1 & 0 & 0 \\
7th day survival & 27 & 7 & 10 & 10 \\
28th day survival & 23 & 5 & 8 & 10 \\
\hline
\end{tabular}

SIRS - systemic inflammatory response syndrome; VD - 25(OH) vitamin D; VD severe deficiency - the plasma level of $25(\mathrm{OH}) \mathrm{D}<12 \mathrm{ng} / \mathrm{mL}$; VD deficiency - the plasma level of $25(\mathrm{OH}) \mathrm{D}<20 \mathrm{ng} / \mathrm{mL}$; VD insufficiency - the plasma level of $25(\mathrm{OH})$ $\mathrm{D}$ between 20-29 ng/mL; VD sufficiency - the plasma level of $25(\mathrm{OH}) \mathrm{D} \geq 30 \mathrm{ng} / \mathrm{mL}$ 
Tab. 2. Comparison of plasma 25(OH)D levels between group of patients with sepsis and group of patients with non-infectious SIRS.

\begin{tabular}{lcccc}
\hline Group & $\mathrm{n}$ & Mean $(\mu \mathrm{g} / \mathrm{L})$ & $\mathrm{SD}$ & $\mathrm{p}$ \\
\hline All patients & 32 & 12.831 & 6.642 & \\
\hline Septic patients & 25 & 11.084 & 4.965 & $0.0032^{*}$ \\
$\quad$ Sepsis & 11 & 14.130 & 3.590 & $0.004^{* *}$ \\
$\quad$ Septic shock & 14 & 8.690 & 4.651 & \\
\hline SIRS & 7 & 19.071 & 8.440 & \\
\hline
\end{tabular}

$\mathrm{n}$ - number, Septic patients - patients with sepsis and septic shock; SD - standard deviation, SIRS - systemic inflammatory response syndrome, $\mathrm{p}$ - double edged ttest, * sepsis vs SIRS, ** sepsis vs septic shock

Chemical Europe GmbH, Dusseldorf, Germany) on an automated immunoassay analyzer. The levels of procalcitonin (PCT) were determined by electrochemiluminescent enzyme immunoassay (ECLEIA), and concentrations of C-reactive protein (CRP) were tested immunoturbidimetricaly. The plasma levels of presepsin, PCT and CRP and routine biochemical and hematological examinations were performed at the specialized biochemical and immunological hospital laboratory Medirex Ltd., Bratislava, Slovakia. In addition, plasma levels of $25(\mathrm{OH}) \mathrm{D}$ were evaluated by electrochemiluminescent binding test (Elecsys Vitamin D total-cobas; Roche Diagnostics GmbH, Mannheim, Germany) in Laboratória Piešt'any Ltd., Piešt'any, Slovakia. All of the parameters and the results mentioned in this study were obtained from samples taken the next day after admission to ICU (within 24 hours - sample T1).

The study is a part of a larger study and was approved by the Ethical Committee for Research of the Faculty of Medicine, Comenius University in Bratislava and University Hospital Bratislava, Slovakia and an informed consent was obtained from each patient or his/her legally authorized representative.

\section{Statistical analysis}

Statistical analysis was performed by the statistical program InStat3.06, GraphPad Software, Inc., San Diego, USA. We compared the values by a two-tailed T-test or non-parametric parametric Mann-Whitney test if the data did not pass the normality test. We also used non-parametric Spearman test for correlation. A significant difference was assumed if $\mathrm{p}$ value was $<0.05$.

\section{Results}

Decreased plasma levels of 25(OH)D in septic patients

Our results from Tab. 1 shows that all sepsis and septic shock patients suffered from $25(\mathrm{OH}) \mathrm{D}$ deficiency (plasma levels of
$25(\mathrm{OH}) \mathrm{D}<20 \mathrm{ng} / \mathrm{mL})$. Out of 7 SIRS patients, 1 had severe 25(OH)D deficiency, 4 had 25(OH)D deficiency and 1 had 25(OH) D insufficiency. Only 1 of SIRS and none of septic patients had normal level of $25(\mathrm{OH}) \mathrm{D}$.

We found significantly decreased levels of $25(\mathrm{OH}) \mathrm{D}$ in all septic (sepsis and septic shock; $\mathrm{n}=25 ; 11.084 \pm 4.965 \mu \mathrm{g} / \mathrm{L}$ ) compared to non-infectious SIRS patients $(\mathrm{n}=7 ; 19.071 \pm 8.440$ $\mu \mathrm{g} / \mathrm{L} ; \mathrm{p}=0.0032$ ) (Tab. 2). We further subdivided the group of septic patients into two subgroups: sepsis $(n=11)$ and septic shock patients $(n=14)$ and found that patients with septic shock had significantly decreased levels of vitamin $\mathrm{D}$ than patients with sepsis $(p=0.004)$ (Tab. 2).

Decreased plasma levels of 25(OH)D in patients, who did not survive the 7th or 28th day of hospital care

Significantly lower levels of $25(\mathrm{OH}) \mathrm{D}$ were found in the group of patients, who did not survive the 7 th day of hospital care $(n=5$; $5.200 \mu \mathrm{g} / \mathrm{L}$; IQR: 7.755) compared to those, who survived the 7th day after admission to ICU ( $=27 ; 14.100 \mu \mathrm{g} / \mathrm{L}$; IQR: 7.20; $\mathrm{p}=$ 0.0076 ) (Tab. 3). A significant difference was found also between the levels of vitamin D in patients, who did not survive the 28th day of hospital care $(\mathrm{n}=8 ; 9.200 \mu \mathrm{g} / \mathrm{L}$; IQR: 9.850) and those, who survived the 28th day of hospital care $(\mathrm{n}=23 ; 13.600 \mu \mathrm{g} / \mathrm{L}$; IQR: 7.200; $\mathrm{p}=0.0338$ ) (Tab. 3).

Plasma levels of inflammatory markers STREM-1, and plasma levels of C-reactive protein, presepsin, and procalcitonin and values of clinical scores in SIRS, all septic patients, and patients with sepsis and septic shock

Our results showed, that septic patients (patients with sepsis and septic shock) had significantly higher levels of CRP ( $p$ $<0.0001)$, presepsin $(\mathrm{p}=0.0017)$, sTREM-1 $(\mathrm{p}=0.0190)$ and PCT $(p=0.0071)$ than non-infectious SIRS patients (Tab. 4). Comparing subdivided groups of septic patients, we found, that the patients suffering from septic shock had significantly higher levels of PCT than patients with sepsis. Other investigated parameters were also higher in septic shock patients than in the patients with sepsis, however the differences were not statistically significant.

Interesting differences were found between the groups of patients with SIRS and sepsis. Patients with sepsis had significantly higher levels of CRP $(p=0.0064)$, sCD14-ST $(p=0.0083)$ and sTREM-1 $(p=0.0379)$, than patients with SIRS.

Tab. 3. Comparison of 25(OH)D levels between group of 7th and 28th day survivors and non-survivors in all of patients with both SIRS and sepsis.

\begin{tabular}{lccc}
\hline Parameter & 7th day non-survivors & 7th day survivors & 28th day non-survivors \\
\hline $\mathrm{n}($ sepsis/SIRS) & $5(5 / 0)$ & $27(20 / 7)$ & $8(7 / 1)$ \\
Min & 3.000 & 3.700 & 3.000 \\
Max & 11.300 & 35.500 & 15.700 \\
Median & 5.200 & 14.100 & 9.200 \\
IQR & 7.750 & 7.200 & 3.700 \\
Mann-Whitney test & & $\mathrm{p}=0.0076$ & 13.600 \\
\hline
\end{tabular}

$\mathrm{n}$ - number, IQR - interquartile range, SIRS - systemic inflammatory response syndrome 
Tab. 4. Plasma levels of modern inflammatory markers in SIRS, all septic patients and subgroups of sepsis and septic shock patients and values of clinical condition scores.

\begin{tabular}{|c|c|c|c|c|}
\hline Parameters & SIRS & Sepsis+Septic shock & Sepsis & Septic shock \\
\hline Number & 7 & 25 & 11 & 14 \\
\hline Mean age (years) & $59.571 \pm 20.759$ & $58.429 \pm 10,525$ & $58.091 \pm 12.565$ & $60.143 \pm 9.330$ \\
\hline Sex (men/women) & $3 / 4$ & $18 / 7$ & $9 / 2$ & $9 / 5$ \\
\hline $\mathrm{CRP}(\mathrm{mg} / \mathrm{L})(\mathrm{mean} \pm \mathrm{SD})$ & $53.016 \pm 34.02$ & $216.624 \pm 124.93$ & $171.867 \pm 111,82$ & $242.574 \pm 138.03$ \\
\hline (T-test) & & $\mathbf{p}<0.0001 *$ & $\mathrm{p}=0.0064^{* *}$ & $\mathrm{p}=0.1813^{* * *}$ \\
\hline $\begin{array}{l}\text { sCD14-ST (ng/L) } \\
\text { (median/IQR/) } \\
\text { (Mann-Whitney) }\end{array}$ & $\begin{array}{c}323 \\
1537 /\end{array}$ & $\begin{array}{c}1355.000 \\
/ 2456.5 / \\
\mathbf{p}=\mathbf{0 . 0 0 1 7}\end{array}$ & $\begin{array}{c}827.000 \\
/ 1210 / \\
\mathbf{p}=\mathbf{0 . 0 0 8 3}\end{array}$ & $\begin{array}{c}2034.000 \\
/ 2752.5 / \\
\mathbf{p}=\mathbf{0 . 0 5 0 7} * * *\end{array}$ \\
\hline $\begin{array}{l}\text { sTREM-1 (ng/L) } \\
\text { (median/IQR/) } \\
\text { (Mann-Whitney) } \\
\end{array}$ & $\begin{array}{c}72,839 \\
/ 36 /\end{array}$ & $\begin{array}{c}131.475 \\
/ 151.8 / \\
\mathbf{p}=\mathbf{0 . 0 1 9 0} *\end{array}$ & $\begin{array}{c}163.420 \\
/ 142.0 / \\
\mathbf{p}=\mathbf{0 . 0 3 7 9} * *\end{array}$ & $\begin{array}{l}120.543 \\
\mathrm{p}= \\
/ 203.8 / \\
0.8429 * * *\end{array}$ \\
\hline $\begin{array}{l}\text { PCT (ng/L) } \\
\quad \text { (median /IQR/) } \\
\text { (Mann-Whitney) }\end{array}$ & $\begin{array}{c}5.07 \\
/ 7.64 /\end{array}$ & $\begin{array}{c}26.021 \\
/ 87.0 / \\
\mathbf{p}=\mathbf{0 . 0 0 7 1} *\end{array}$ & $\begin{array}{c}20.580 \\
152.8 / \\
\mathrm{p}=0.2463^{* *}\end{array}$ & $\begin{array}{c}95.910 \\
/ 80.5 / \\
\mathbf{p}=\mathbf{0 . 0 1 9 8} * * *\end{array}$ \\
\hline SOFA (mean \pm SD) & $11.429 \pm 2.573$ & $13.063 \pm 2.154$ & $13.182 \pm 1.940$ & $13.786 \pm 1.762$ \\
\hline APACHE II (mean \pm SD) & $22.571 \pm 10.937$ & $34.120 \pm 7.102$ & $31.818 \pm 7.278$ & $35.928 \pm 6.662$ \\
\hline
\end{tabular}

Tab. 5. Correlation between the levels of 25(OH)D and inflammatory markers/clinical scores in all of patients with both infectious and noninfectious systemic inflammatory response syndrome (sepsis+noninfectious SIRS).

\begin{tabular}{lcccccc}
\hline $25(\mathrm{OH}) \mathrm{D}$ & CRP & sCD14-ST & sTREM-1 & PCT & SOFA & APACHE II \\
\hline $\mathrm{n}$ & 32 & 32 & 27 & 32 & 32 & -0.2813 \\
\hline $\mathrm{SR}$ & -0.6012 & -0.5045 & -0.5110 & -0.3190 & -0.3676 & -0.5810 \\
\hline $95 \%$ CI & -0.7894 & -0.7307 & -0.7514 & -0.6077 & -0.6413 & -0.01084 \\
\hline $\mathrm{p}$ & -0.3096 & -0.1786 & -0.1509 & 0.04425 & 0.08551 \\
\hline
\end{tabular}

$\mathrm{n}$-number; SR - Spearman r; CI - confidence interval; sTREM-1 - soluble triggering receptor expressed on myeloid cells; sCD14-ST - presepsin; PCT - procalcitonin; SOFA - sequential organ failure assessment score; APACHE II - acute physiology and chronic health evaluation II

Correlation between the levels of 25(OH)D and levels of inflammatory markers STREM-1, C-reactive protein, presepsin, procalcitonin and values of clinical condition scores

We revealed a very significant negative correlations between the levels of 25(OH)D and inflammatory markers CRP ( $p$ $=0.0003), \operatorname{sCD} 14-\mathrm{ST}(\mathrm{p}=0.0032), \mathrm{sTREM}-1(\mathrm{p}=0.0065)$ and $\mathrm{a}$ modest non-significant negative correlation with levels of PCT $(\mathrm{p}=$ 0.0752) in all of the patients with systemic inflammatory response of both infectious and non-infectious origin (Tab. 5).

There was also a significant correlation between the $25(\mathrm{OH})$ D levels and clinical condition of patients represented by SOFA score $(p=0.0385)$ in all of the patients, but not with APACHE II score $(\mathrm{p}=0.1188)($ Tab. 5).

\section{Discussion}

VD deficiency is common among the general population. It is also observed in up to $76 \%$ of critically ill patients (41). Despite the high prevalence of hypovitaminosis D in critical illness, VD is often overlooked by medical staff as the clinical implications and consequences of VD deficiency in acute contexts remain to be fully understood (42).
Both SIRS and sepsis in their beginnings are predominantly associated with an exaggerated inflammatory response and elevated inflammatory markers. That is why the mortality of sepsis in early phase of this disease is associated with hyperinflammation and failure of vital organs due to cytokine storm. During later phase of sepsis, the contra-regulatory immune response, that downregulate the inflammation, can lead to immune suppression, even immune paralysis, what is very dangerous for patients that can die due to polymicrobial sepsis $(18,43,44)$.

To reduce the mortality, the early diagnosis of sepsis (SIRS with proven infectious agent) is essential, and the knowledge about immune status of patients is also very important. Early diagnosis of bacteremia is extremely important for the implementation of antimicrobial therapy. Although blood culture is the "gold standard" for diagnosis of bacteremia, this method has limited usefulness for the early detection of blood-stream infection. Some inflammatory biomarkers - CRP, sTREM-1, HMGB1 (high mobility group box 1) and presepsin are studied as predictors of bacteremia in SIRS/ sepsis patients. Also, for SIRS/sepsis monitoring, the state of immunity (Th1 vs Th2) and the levels of inflammatory and antiinflammatory molecules and cytokines that might be influenced by many factors, are useful to test. 


\section{4-290}

In this part of our study, we decided to analyze the plasma levels of 25(OH)D in SIRS, sepsis and septic shock patients, and find an association with 7th and 28th days mortality. Moreover, we were interested also in correlation of the plasma levels of vitamin D with levels of CRP and modern inflammatory markers presepsin, sTREM-1 and PCT, their difference between SIRS and sepsis and finally the correlation between the levels of 25(OH)D with tested inflammatory markers.

Presepsin, the soluble CD14 subtype, is a cleavage product of the $55 \mathrm{kDa}$ membrane CD14 (mCD14), that colocalizes with toll like receptor 4 (TLR4) and upon binding of the lipopolysaccharide - lipopolysaccharide binding protein complex (LPBP) to this receptor, CD14 activates the TLR4/MD2 - specific pro-inflammatory signaling cascade, thereby starting the inflammatory reaction of the host against infectious agents (45). The complex of LPBP-CD14 is released into circulation by shedding of CD14 from the cell membrane yielding soluble CD14 (sCD14). Another soluble sCD14-ST - presepsin, a $13 \mathrm{kDa}$ fragment derived from cleavage of $\mathrm{mCD} 14$ can be released during phagocytosis and cleavage with plasma proteases, lysosomal enzymes and cathepsin D (46). Presepsin is used as an aid in the diagnosis and prognosis of sepsis, in the assessment of the degree of septic severity and to aid in the risk stratification of critically ill septic patients. It may contribute to rule out the diagnosis of bacteremia in SIRS patients admitted to the Emergency Department (47) and seems to provide better early diagnostic value than procalcitonin in early diagnosis of neonatal sepsis (48).

TREM-1 belongs to one of the newer PRRs, however its ligand is still unknown. It was first described in 2000 on the surface of myeloid cells (49). It is expressed on the surface of neutrophils, mature monocytes, macrophages and non-myeloid cells, such as epithelial and endothelial cells $(50,51)$, has pro-inflammatory activity $(49,52)$ and the presence of extracellular bacteria, fungi and their products increase its expression. The extracellular domain of TREM-1 can be cleaved and released in the body fluids as a soluble TREM-1 (sTREM-1) (53), moreover, it can be also produced by cells (mainly activated monocytes and macrophages) and functions as a decoy receptor present in different body fluids. The levels of sTREM-1 can be measured by ELISA and can serve as a diagnostic inflammatory marker (54-57). Both TREM-1 and sTREM-1 play a great role in the inflammatory response regulation. Increased TREM-1 expression and increased levels of sTREM-1 accompany both infectious and non-infectious inflammatory processes $(54,56)$, but more intensively infectious processes.

The mean level of $25(\mathrm{OH}) \mathrm{D}(12.831 \pm 6.642 \mu \mathrm{g} / \mathrm{L})$ in the examined cohort of 32 adult patients admitted to the ICU because of systemic inflammatory response syndrome of both infectious and non-infectious origin is deeply below the normal range of $25(\mathrm{OH}) \mathrm{D}$ level $(>30 \mu \mathrm{g} / \mathrm{L})(37,39,40)$. It is even approaching the boundary of severe vitamin D deficiency $(25(\mathrm{OH}) \mathrm{D}$ level < $12 \mu \mathrm{g} / \mathrm{L})(58,59)$.

The finding of significantly decreased levels of $25(\mathrm{OH}) \mathrm{D}$ in the group of septic patients $(11.084 \pm 4.965 \mu \mathrm{g} / \mathrm{L})$ compared to group of non-infectious SIRS patients $(\mathrm{p}=0.0032)$ corresponds with the results of mentioned studies supporting the role of VD in both the innate and adaptive immune responses to viral and bacterial infections $(25,26)$ and the association between low vitamin $\mathrm{D}$ levels and risk of sepsis $(60,61)$.

The significantly lower levels of $25(\mathrm{OH}) \mathrm{D}$ ranking in severe VD deficiency range in the group of patients, who did not survive the 7th day of hospital care compared to those, who survived the 7 th day after admission to ICU $(p=0.0076)$, point out the potential impact of VD level on early mortality risk in patients with systemic inflammatory response syndrome of both SIRS and sepsis. This finding consents with the results of other authors who declare, that VD deficiency at the time of critical care initiation is a significant predictor of overall cause patient mortality in a critically ill patient population, independently of other comorbidities $(59,62)$. This also correspond with our significant correlation with SOFA score, but non-significant correlation with APACHE II - a scoring system of clinical condition of patients, that takes into account also other comorbidities, not only acute state.

Our results showed, that septic patients had significantly higher levels of CRP $(p<0.0001)$, presepsin $(p=0.0017)$, sTREM-1 ( $p$ $=0.0190)$ and PCT $(p=0.0071)$, than non-infectious SIRS patients (Tab. 4). Comparing subdivided group of septic patients, we found, that patients suffering from septic shock had significantly higher levels of PCT than patients with sepsis. Interesting differences were found between the groups of patients with SIRS and sepsis. Patients with sepsis had significantly higher levels of CRP $(p=0.0064)$, sCD14-ST $(p=0.0083)$ and sTREM-1 $(p=0.0379)$, than patients with SIRS, so these markers might serve as diagnostic markers between SIRS and sepsis, that we try to prove in a larger cohort of patients.

Besides the reduced immunological defense against infection in VD deficient septic patients, insufficient regulatory and anti-inflammatory mechanisms of the immune system due to VD deficiency in both patient groups (sepsis and SIRS) can cause more intense and stormy early pro-inflammatory Th1 response of systemic inflammatory response syndrome with a higher lethality. This hypothesis is also supported by our findings of significant negative correlation between the levels of $25(\mathrm{OH}) \mathrm{D}$ and inflammatory markers like CRP $(p=0.0003), \operatorname{sCD} 14-S T(p=0.0032)$ and modest non-significant negative correlation with PCT levels $(\mathrm{p}=0.0752)$ in all SIRS/sepsis patients. Very significant negative correlation was also detected between the levels of 25(OH)D and sTREM-1 $(p=0.0065)$ in all of the patients.

VD status is associated with adverse outcomes in the critically ill. Despite, there are only a few clinical studies that evaluated VD supplementation in critically ill patients. Amrein et al (58) conducted the largest randomized controlled trial to date investigating the influence of a high-dose bolus enteral vitamin D3 supplementation on outcomes of 475 critically ill medical and surgical adult patients with VD deficiency $(\leq 20 \mathrm{ng} / \mathrm{mL})$. The authors concluded that a high-dose vitamin D3 did not reduce hospital length of stay, hospital mortality, or 6-month mortality. However, they observed a lower hospital mortality in patients with severe VD deficiency $(\leq 12 \mathrm{ng} / \mathrm{mL})$ at baseline. A systematic review and meta-analysis of 7 randomized controlled trials (716 patients) concluded that VD administration was associated with a decreased mortality in 
critically ill patients without serious adverse events (63). Clinical studies demonstrate that 1,25-dihydroxyvitamin D (1,25(OH)2D3) protects patients from adverse outcome of sepsis, but clinical treatment with $1,25(\mathrm{OH}) 2 \mathrm{D} 3$ is rare.

In 2018 Rao et al performed a clinical study on animal model and found that $1,25(\mathrm{OH}) 2 \mathrm{D} 3$ treatment had beneficial effects and improved the survival rate in LPS-induced mouse model of sepsis by blocking the secretion of HMGB1, the key late regulator of sepsis. LPS-induced HMGB1 secretion was attenuated by $1,25(\mathrm{OH}) 2 \mathrm{D} 3$ via blocking HMGB1 translocation from the nucleus to the cytoplasm in macrophages. $1,25(\mathrm{OH}) 2 \mathrm{D} 3 \mathrm{can}$ induce the expression of hemeoxygenase-1 (HO-1), which is essential for blocking HMBG1 nuclear translocation and its secretion. $1,25(\mathrm{OH}) 2 \mathrm{D} 3$ is recognized as the key mediator of inflammatory diseases, including sepsis (64). The authors provide evidence that 1,25(OH)2D3 attenuates LPS-induced HMGB1 secretion via the $\mathrm{Nrf} 2$ (nuclear factor erythroid 2-related factor 2)/HO-1 pathway in macrophages.

\section{Conclusion}

Our results showed that except one all tested ICU (SIRS/sepsis) patients had decreased or insufficient levels of plasma VD with the lowest levels in septic shock patients. The lower levels of $25(\mathrm{OH}) \mathrm{D}$ in the group of 7 th and 28th day non-survivors point out the potential impact of VD level on early mortality risk and support the assumption that VD deficiency at the time of critical care initiation is a significant predictor of mortality. The levels of inflammatory markers CRP, presepsin, sTREM-1 negatively correlated with the plasma levels of VD, what indicates, that VD deficiency can cause more intense and stormy early pro-inflammatory Th1 response with a higher lethality. This hypothesis is also supported by our findings of a negative correlation between the levels of $25(\mathrm{OH}) \mathrm{D}$ and SOFA score.

\section{References}

1. Geroulanos S, Douka ET. Historical perspective of the word "sepsis". Intensive Care Med 2006; 32 (12): 2077.

2. Singer M, Deutschman CS, Seymour CW, Shankar-Hari M, Annane D, Bauer $M$ et al. The Third International Consensus Definitions for Sepsis and Septic Shock (Sepsis-3). JAMA 2016; 315 (8): 801-810.

3. Gaieski DF, Edwards JM, Kallan MJ, Carr BG. Benchmarking the incidence and mortality of severe sepsis in the United States. Crit Care Med 2013; 41 (5): 1167-1174.

4. Fleischmann C, Scherag A, Adhikari NK, Hartog CS, Tsaganos T, Schlattmann P et al. Assessment of Global Incidence and Mortality of Hospital-treated Sepsis. Current Estimates and Limitations. Am J Respir Crit Care Med 2016; 193 (3): 259-272.

5. Vincent JL, Marshall JC, Namendys-Silva SA, Francois B, MartinLoeches I, Lipman J et al. Assessment of the worldwide burden of critical illness: the intensive care over nations (ICON) audit. Lancet Respir Med 2014; 2 (5): 380-386.

6. Brun-Buisson C. The epidemiology of the systemic inflammatory response. Intensive Care Med 2000; 26 Suppl 1: S64-74.
7. Caserta S, Mengozzi M, Kern F, Newbury SF, Ghezzi P, Llewelyn MJ. Severity of Systemic Inflammatory Response Syndrome Affects the Blood Levels of Circulating Inflammatory-Relevant MicroRNAs. Front Immunol 2017; 8: 1977.

8. Davies MG, Hagen PO. Systemic inflammatory response syndrome. $\mathrm{Br}$ J Surg 1997; 84 (7): 920-935.

9. Parlato M, Cavaillon JM. Host response biomarkers in the diagnosis of sepsis: a general overview. Methods Mol Biol 2015; 1237: 149-211.

10. Hayashi T, Nakamura T, Takaoka A. [Pattern recognition receptors]. Nihon Rinsho Meneki Gakkai Kaishi 2011; 34 (5): 329-345.

11. Leijte GP, Custers H, Gerretsen J, Heijne A, Roth J, Vogl T et al. Increased Plasma Levels of Danger-Associated Molecular Patterns Are Associated With Immune Suppression and Postoperative Infections in Patients Undergoing Cytoreductive Surgery and Hyperthermic Intraperitoneal Chemotherapy. Front Immunol 2018; 9: 663.

12. Lamkanfi M, Dixit VM. Mechanisms and functions of inflammasomes. Cell 2014; 157 (5): 1013-1022.

13. Opitz B, van Laak V, Eitel J, Suttorp N. Innate immune recognition in infectious and noninfectious diseases of the lung. Am J Respir Crit Care Med 2010; 181 (12): 1294-1309.

14. Schaefer L. Complexity of danger: the diverse nature of damage-associated molecular patterns. J Biol Chem 2014; 289 (51): 35237-35245.

15. Andersson U, Yang H, Harris H. High-mobility group box 1 protein (HMGB1) operates as an alarmin outside as well as inside cells. Semin Immunol 2018; 38: 40-48.

16. Chousterman BG, Swirski FK, Weber GF. Cytokine storm and sepsis disease pathogenesis. Semin Immunopathol 2017; 39 (5): 517-528.

17. Makhija R, Kingsnorth AN. Cytokine storm in acute pancreatitis. J Hepatobiliary Pancreat Surg 2002; 9 (4): 401-410.

18. Sauaia A, Moore FA, Moore EE. Postinjury Inflammation and Organ Dysfunction. Crit Care Clin 2017; 33 (1): 167-191.

19. Vincent JL, de Mendonca A, Cantraine F, Moreno R, Takala J, Suter PM et al. Use of the SOFA score to assess the incidence of organ dysfunction/failure in intensive care units: results of a multicenter, prospective study. Working group on "sepsis-related problems" of the European Society of Intensive Care Medicine. Crit Care Med 1998; 26 (11): 1793-1800.

20. Knaus WA, Draper EA, Wagner DP, Zimmerman JE. APACHE II: a severity of disease classification system. Crit Care Med 1985; 13 (10): 818-829.

21. Adams JS, Hewison M. Update in vitamin D. J Clin Endocrinol Metab 2010; 95 (2): 471-478.

22. Bivona G, Agnello L, Ciaccio M. The immunological implication of the new vitamin D metabolism. Cent Eur J Immunol 2018; 43 (3): 331-334.

23. Holick MF. Vitamin D: importance in the prevention of cancers, type 1 diabetes, heart disease, and osteoporosis. Am J Clin Nutr 2004; 79 (3): 362-371.

24. Holick MF. Vitamin D deficiency. N Engl J Med 2007; 357 (3): 266-281.

25. Beard JA, Bearden A, Striker R. Vitamin D and the anti-viral state. J Clin Virol 2011; 50 (3): 194-200.

26. Bikle DD. Vitamin D and the immune system: role in protection against bacterial infection. Curr Opin Nephrol Hypertens 2008; 17 (4): 348-352.

27. Mahon BD, Wittke A, Weaver V, Cantorna MT. The targets of vitamin $\mathrm{D}$ depend on the differentiation and activation status of $\mathrm{CD} 4$ positive $\mathrm{T}$ cells. J Cell Biochem 2003; 89 (5): 922-932.

28. Veldman CM, Cantorna MT, DeLuca HF. Expression of 1,25-dihydroxyvitamin $\mathrm{D}(3)$ receptor in the immune system. Arch Biochem Biophys 2000; 374 (2): 334-338.

29. Yu XP, Bellido T, Manolagas SC. Down-regulation of NF-kappa B protein levels in activated human lymphocytes by 1,25-dihydroxyvitamin D3. Proc Natl Acad Sci U S A 1995; 92 (24): 10990-10994. 
30. Hewison M. Vitamin D and the immune system: new perspectives on an old theme. Endocrinol Metab Clin North Am 2010; 39 (2): 365-379, table of contents.

31. Lemire JM, Archer DC, Beck L, Spiegelberg HL. Immunosuppressive actions of 1,25-dihydroxyvitamin D3: preferential inhibition of Th1 functions. J Nutr 1995; 125 (6 Suppl): 1704S-1708S.

32. Colotta F, Jansson B, Bonelli F. Modulation of inflammatory and immune responses by vitamin D. J Autoimmun 2017; 85: 78-97.

33. Vanherwegen AS, Gysemans C, Mathieu C. Regulation of Immune Function by Vitamin D and Its Use in Diseases of Immunity. Endocrinol Metab Clin North Am 2017; 46 (4): 1061-1094

34. Akdere G, Efe B, Sisman P, Yorulmaz G. The relationship between vitamin D level and organspecific autoimmune disorders in newly diagnosed type I diabetes mellitus. Bratisl Lek Listy 2018; 119 (9): 544-549.

35. Lee P, Nair P, Eisman JA, Center JR. Vitamin D deficiency in the intensive care unit: an invisible accomplice to morbidity and mortality? Intensive Care Med 2009; 35 (12): 2028-2032.

36. Sebekova K, Krivosikova Z, Gajdos M, Podracka L. Vitamin D status in apparently healthy medication-free Slovaks: Association to blood pressure, body mass index, self-reported smoking status and physical activity. Bratisl Lek Listy 2016; 117 (12): 702-709.

37. Holick MF. High prevalence of vitamin D inadequacy and implications for health. Mayo Clin Proc 2006; 81 (3): 353-373.

38. Holick MF. Resurrection of vitamin D deficiency and rickets. J Clin Invest 2006; 116 (8): 2062-2072.

39. Bischoff-Ferrari HA, Giovannucci E, Willett WC, Dietrich T, DawsonHughes B. Estimation of optimal serum concentrations of 25-hydroxyvitamin D for multiple health outcomes. Am J Clin Nutr 2006; 84 (1): 18-28.

40. Holick MF. Vitamin D status: measurement, interpretation, and clinical application. Ann Epidemiol 2009; 19 (2): 73-78.

41. Dickerson RN, Van Cleve JR, Swanson JM, Maish GO, 3rd, Minard G, Croce MA et al. Vitamin D deficiency in critically ill patients with traumatic injuries. Burns Trauma 2016; 4: 28.

42. Al-Tarrah K, Hewison M, Moiemen N, Lord JM. Vitamin D status and its influence on outcomes following major burn injury and critical illness. Burns Trauma 2018; 6: 11.

43. Wang H, Ma S. The cytokine storm and factors determining the sequence and severity of organ dysfunction in multiple organ dysfunction syndrome. Am J Emerg Med 2008; 26 (6): 711-715.

44. Gentile LF, Cuenca A, Efron P, Ang D, Bihorac A, McKinley B et al. Persistent inflammation and immunosuppression: a common syndrome and new horizon for surgical intensive care. J Trauma Acute Care Surg 2012; 72 (6; 2163-0763 (Electronic)): 1491-1501.

45. Ryu JK, Kim SJ, Rah SH, Kang JI, Jung HE, Lee D et al. Reconstruction of LPS Transfer Cascade Reveals Structural Determinants within LBP, CD14, and TLR4-MD2 for Efficient LPS Recognition and Transfer. Immunity 2017; 46 (1): 38-50.

46. Chenevier-Gobeaux C, Borderie D, Weiss N, Mallet-Coste T, Claessens YE. Presepsin (sCD14-ST), an innate immune response marker in sepsis. (1873-3492 (Electronic)).

47. Romualdo LG, Torrella PE, Gonzalez MV, Sanchez RJ, Holgado AH, Freire AO et al. Diagnostic accuracy of presepsin (soluble CD14 subtype) for prediction of bacteremia in patients with systemic inflammatory response syndrome in the Emergency Department. Clin Biochem 2014; 47 (7-8): 505-508.

48. Iskandar A, Arthamin MZ, Indriana K, Anshory M, Hur M, Di Somma S. Comparison between presepsin and procalcitonin in early diagnosis of neonatal sepsis. J Matern Fetal Neonatal Med 2018; (1476-4954 (Electronic)): [Epub ahead of print].
49. Bouchon A, Dietrich J, Colonna M. Cutting edge: inflammatory responses can be triggered by TREM-1, a novel receptor expressed on neutrophils and monocytes. J Immunol 2000; 164 (10): 4991-4995.

50. Gingras MC, Lapillonne H, Margolin JF. TREM-1, MDL-1, and DAP12 expression is associated with a mature stage of myeloid development. Mol Immunol 2002; 38 (11): 817-824.

51. Rigo I, McMahon L, Dhawan P, Christakos S, Yim S, Ryan LK et al. Induction of triggering receptor expressed on myeloid cells (TREM-1) in airway epithelial cells by $1,25(\mathrm{OH})(2)$ vitamin $\mathrm{D}(3)$. Innate Immun 2012; 18 (2): 250-257.

52. Bouchon A, Facchetti F, Weigand MA, Colonna M. TREM-1 amplifies inflammation and is a crucial mediator of septic shock. Nature 2001; 410 (6832): 1103-1107.

53. Gomez-Pina V, Soares-Schanoski A, Rodriguez-Rojas A, Del Fresno C, Garcia F, Vallejo-Cremades MT et al. Metalloproteinases shed TREM1 ectodomain from lipopolysaccharide-stimulated human monocytes. J Immunol 2007; 179 (6): 4065-4073.

54. Bucova M, Suchankova M, Dzurilla M, Vrlik M, Novosadova H, Tedlova $\mathbf{E}$ et al. Inflammatory marker sTREM-1 reflects the clinical stage and respiratory tract obstruction in allergic asthma bronchiale patients and correlates with number of neutrophils. Mediators Inflamm 2012; 2012: 628754.

55. Suchankova M, Bucova M, E T, Demian J, Majer I, Novosadova H et al. sTREM-1 in bronchoalveolar lavage fluid in patients with pulmonary sarcoidosis, effect of smoking and inflammation. Bratisl Lek Listy 2013; 114 (12): 702-707.

56. Gibot S, Cravoisy A. Soluble form of the triggering receptor expressed on myeloid cells-1 as a marker of microbial infection. Clin Med Res 2004; 2 (3): 181-187.

57. Pelham CJ, Pandya AN, Agrawal DK. Triggering receptor expressed on myeloid cells receptor family modulators: a patent review. Expert Opin Ther Pat 2014; 24 (12): 1383-1395.

58. Amrein K, Schnedl C, Holl A, Riedl R, Christopher KB, Pachler C et al. Effect of high-dose vitamin D3 on hospital length of stay in critically ill patients with vitamin D deficiency: the VITdAL-ICU randomized clinical trial. JAMA 2014; 312 (15): 1520-1530.

59. Trongtrakul K, Feemuchang C. Prevalence and association of vitamin $\mathrm{D}$ deficiency and mortality in patients with severe sepsis. Int $\mathrm{J}$ Gen Med 2017; 10: 415-421.

60. Jovanovich AJ, Ginde AA, Holmen J, Jablonski K, Allyn RL, Kendrick $\mathbf{J}$ et al. Vitamin D level and risk of community-acquired pneumonia and sepsis. Nutrients 2014; 6 (6): 2196-2205.

61. Moromizato T, Litonjua AA, Braun AB, Gibbons FK, Giovannucci E, Christopher KB. Association of low serum 25-hydroxyvitamin D levels and sepsis in the critically ill. Crit Care Med 2014; 42 (1): 97-107.

62. Braun AB, Gibbons FK, Litonjua AA, Giovannucci E, Christopher KB. Low serum 25-hydroxyvitamin $\mathrm{D}$ at critical care initiation is associated with increased mortality. Crit Care Med 2012; 40 (1): 63-72.

63. Putzu A, Belletti A, Cassina T, Clivio S, Monti G, Zangrillo A et al. Vitamin D and outcomes in adult critically ill patients. A systematic review and meta-analysis of randomized trials. J Crit Care 2017; 38: 109-114.

64. Rao Z, Zhang N, Xu N, Pan Y, Xiao M, Wu J et al. Corrigendum: 1,25-Dihydroxyvitamin D Inhibits LPS-Induced High-Mobility Group Box 1 (HMGB1) Secretion via Targeting the NF-E2-Related Factor 2-Hemeoxygenase-1-HMGB1 Pathway in Macrophages. Front Immunol 2018; 9: 357.

Received January 28, 2019. Accepted March 1, 2019. 